\begin{tabular}{|c|l|}
\hline Title & Breathing multichimera states in nonlocally coupled phase oscillators \\
\hline Author(s) & Suda, Y usuke; Okuda, Koji \\
\hline Citation & $\begin{array}{l}\text { Physical Review E, 97(4), 042212 } \\
\text { https://doi.org/10.1103/PhysRevE.97.042212 }\end{array}$ \\
\hline Issue Date & 2018-0419 \\
\hline Doc URL & http://hdl.handle.net/2115/71001 \\
\hline Rights & @2018A merican Physical Society \\
\hline Type & article \\
\hline File Information & PhysRevE.97.042212.pdf \\
\hline
\end{tabular}

Instructions for use 


\title{
Breathing multichimera states in nonlocally coupled phase oscillators
}

\author{
Yusuke Suda and Koji Okuda \\ Division of Physics, Hokkaido University, Sapporo 060-0810, Japan
}

(Received 28 September 2017; revised manuscript received 22 February 2018; published 19 April 2018)

\begin{abstract}
Chimera states for the one-dimensional array of nonlocally coupled phase oscillators in the continuum limit are assumed to be stationary states in most studies, but a few studies report the existence of breathing chimera states. We focus on multichimera states with two coherent and incoherent regions and numerically demonstrate that breathing multichimera states, whose global order parameter oscillates temporally, can appear. Moreover, we show that the system exhibits a Hopf bifurcation from a stationary multichimera to a breathing one by the linear stability analysis for the stationary multichimera.
\end{abstract}

DOI: 10.1103/PhysRevE.97.042212

\section{INTRODUCTION}

Coupled oscillator systems have been studied extensively in various scientific fields for many years [1,2]. In particular, chimera states of coupled oscillators have recently attracted great interest [3-34]. Chimera states can be seen in a wide variety of systems with different coupling topologies $[4,8,9,11,17,22,28,29,32]$ and different kinds of constituent oscillators [14,16,17,20,22,28-30], and are also found experimentally $[18,19,22,25]$. One of the most basic models among them is the one-dimensional array of nonlocally coupled phase oscillators $[3,5-7,10,12,13,15,21,23,24,26,31,33,34]$. The essential feature of chimera states in phase oscillator systems on one-dimensional space is the coexistence of coherent regions of synchronized oscillators and incoherent regions of drifting oscillators. The morphology of their coexistence depends on the coupling function corresponding to the interaction between oscillators and the coupling kernel function characterizing nonlocality. In many cases, only the fundamental harmonic component is used for the coupling function, as in the present paper. However, it is also reported that higher harmonic components in the coupling function are responsible for a rich variety of chimera states [26,31,34].

Chimera states for the one-dimensional array of phase oscillators in the continuum limit $N \rightarrow \infty$, where $N$ is the number of oscillators, are mostly assumed to be stationary states. This means that the local mean field or the local order parameter is stationary in the rotating frame with a constant frequency. This assumption about chimera states plays an important role in various studies, e.g., the self-consistency equation of the local mean field [3,5-7] and the linear stability analysis of chimera states [21,24,33]. However, Abrams et al. [8] discovered breathing chimeras, whose global order parameter of a population oscillates temporally, for two interacting populations of globally coupled phase oscillators, and posed the question of whether such breathing chimeras exist in the case of one-dimensional arrays. To answer this question, Laing [10] demonstrated that there also appear breathing chimeras in the one-dimensional system by introducing phase lag parameter heterogeneity.

In this paper, we focus on chimera states, especially multichimera states with two coherent and incoherent regions, in one-dimensional nonlocally coupled phase oscillators. Moreover, it is demonstrated that breathing chimeras can appear even in homogeneous systems without introducing parameter heterogeneity. By numerical simulations, we observe that the appearance of breathing chimeras depends on the coupling kernel function. Then we show that the system exhibits a Hopf bifurcation from a stationary chimera to a breathing one by the linear stability analysis for the stationary chimera.

\section{MODEL}

We consider the system of nonlocally coupled phase oscillators obeying

$$
\dot{\theta}(x, t)=\omega-\int_{-\pi}^{\pi} d y G(x-y) \sin [\theta(x, t)-\theta(y, t)+\alpha],
$$

with $2 \pi$-periodic phase $\theta(x, t)$ on the one-dimensional space $x \in[-\pi, \pi]$ under the periodic boundary condition. The coupling between oscillators is assumed to be the sine function with the phase lag parameter $\alpha$ [35], and the natural frequency $\omega$ can be set to zero without loss of generality. The coupling kernel function $G(x)$ is generally an even real function described as

$$
G(x)=\sum_{k=0}^{\infty} g_{k} \cos (k x),
$$

where $g_{k} \in \mathbb{R}$ and $x \in[-\pi, \pi]$. Nonlocal coupling is characterized by this kernel, which can be taken as, e.g., the exponential kernel $[3,7,33,34]$ and the cosine kernel $[5,6,10,24]$. In this paper, we particularly use the step kernel $[12,13,15,21,23,31]$,

$$
G(x)= \begin{cases}1 /(2 \pi r) & (|x| \leqslant \pi r) \\ 0 & (|x|>\pi r),\end{cases}
$$

with $0<r \leqslant 1$, where $r$ denotes the coupling range. For numerical simulation of Eq. (1), we need to discretize $x$ into $x_{j}:=-\pi+2 \pi j / N(j=0, \ldots, N-1)$. Then Eq. (1) with the step kernel given by Eq. (3) is rewritten as

$$
\dot{\theta}_{j}(t)=\omega-\frac{1}{2 R} \sum_{k=j-R}^{j+R} \sin \left[\theta_{j}(t)-\theta_{k}(t)+\alpha\right],
$$



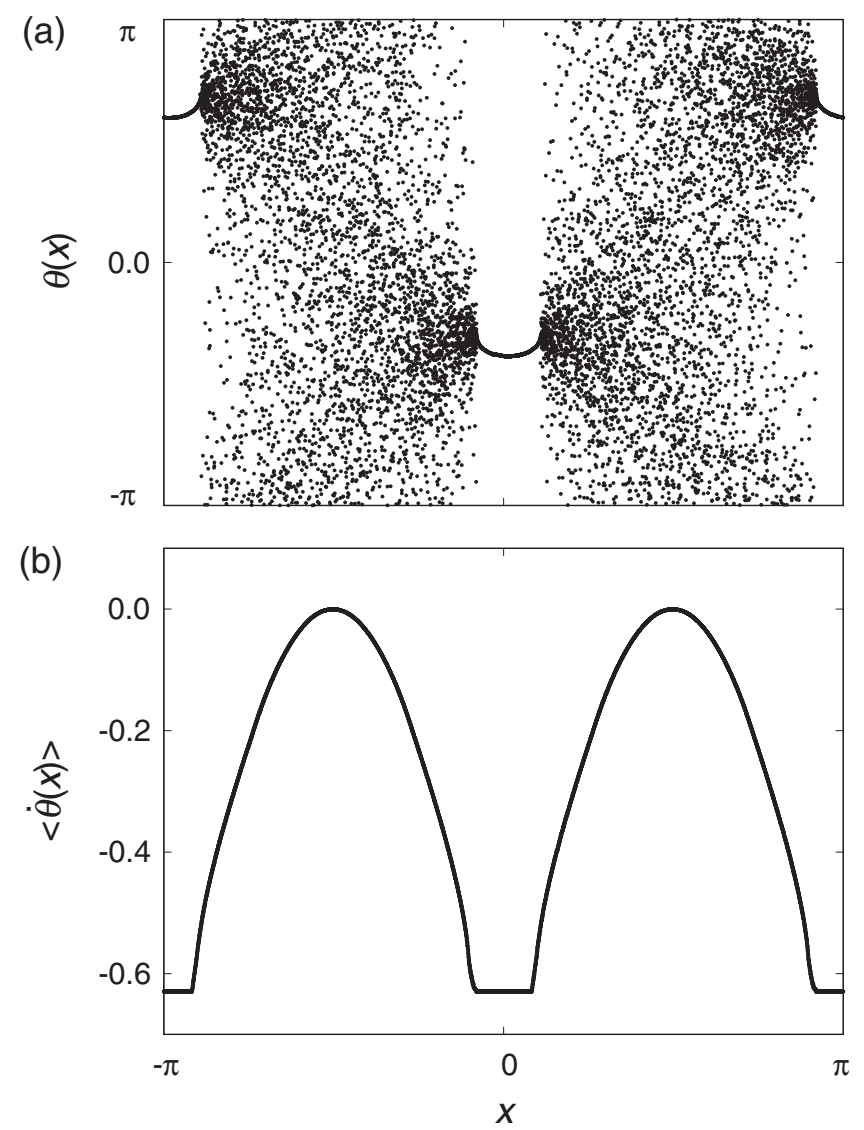

FIG. 1. Multichimera state with two coherent and incoherent regions for Eq. (4) with $N=10000, \alpha=1.480$, and $r=0.360$. (a) The snapshot of the phase $\theta(x, t)$. (b) The profile of the average frequency $\langle\dot{\theta}(x)\rangle$ with $T=2000$.

where $\theta_{j}(t):=\theta\left(x_{j}, t\right)$ and $R:=r N / 2$. All the indexes in Eq. (4) are regarded as modulo $N$, considering the periodic boundary condition. In this paper, we use the fourth-order Runge-Kutta method with time interval $\Delta t=0.01$ for all numerical simulations. Note that chimera states for Eq. (4) are merely transient for small $N$, but, when $N$ is larger, the transient time becomes longer and diverges to infinity in the continuum limit, where the chimera state appears stable $[13,15,25,31]$.

For Eq. (4), there appear various types of chimera states [23], including multichimera states with two or more coherent and incoherent regions. A typical multichimera state obtained by numerical simulation is shown in Fig. 1. This multichimera state has two coherent and incoherent regions, which we call 2-multichimera below. Figure 1(a) shows the snapshot of the phase $\theta(x, t)$. Two coherent regions are separated from each other by the phase almost exactly $\pi$, which is a remarkable feature of the 2-multichimera and different from merely two neighboring chimeras. In order to assist the emergence of 2-multichimera for the numerical simulations of Eq. (4), we use the following initial condition close to a 2-multichimera [6,31]:

$$
\theta(x)= \begin{cases}\exp \left[-30\left(\frac{|x|}{2 \pi}-\frac{1}{4}\right)^{2}\right] p(x) & \left(0 \leqslant|x| \leqslant \frac{\pi}{2}\right) \\ \exp \left[-30\left(\frac{|x|}{2 \pi}-\frac{1}{4}\right)^{2}\right] p(x)+\pi & \left(\frac{\pi}{2}<|x| \leqslant \pi\right),\end{cases}
$$

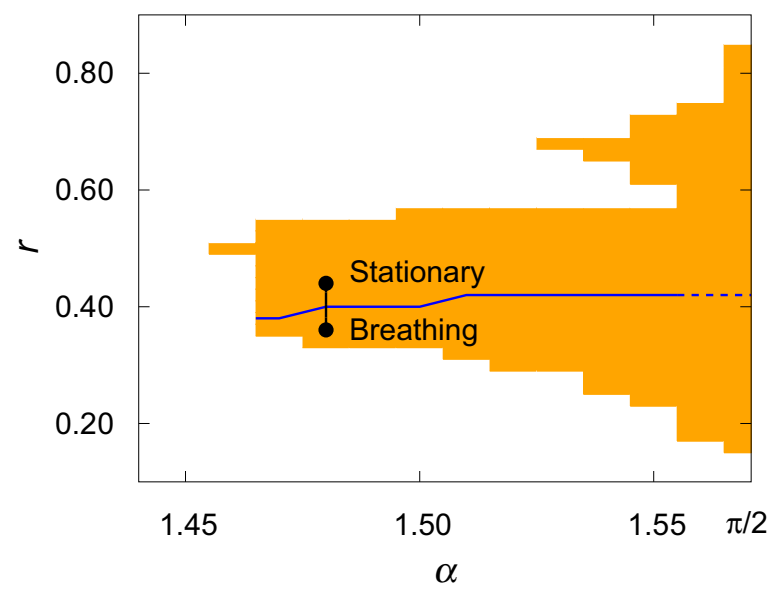

FIG. 2. Stability region of 2-multichimera for Eq. (4) obtained by the numerical simulation with $N=100000$. Black circles denote the parameter values of Fig. 3. The blue line denotes the Hopf bifurcation points obtained by the linear stability analysis for the stationary 2-multichimera with fixed $\alpha$ in Sec. IV, but we could not determine those points for $\alpha$ close to $\pi / 2$ (dashed line).

where $p(x) \in[-\pi, \pi]$ is a uniform random number. Figure 1(b) shows the profile of the average frequency $\langle\dot{\theta}(x)\rangle:=\int_{0}^{T} d t^{\prime} \dot{\theta}\left(x, t^{\prime}\right) / T$ with the measurement time $T$. When we refer to time-averaged quantities $\langle\cdot\rangle$, we set the measurement time to $T=2000$ and measured those quantities after the transient time 2000. The stability region of the 2-multichimera for Eq. (4) obtained by the numerical simulation with $N=100000$ is shown in Fig. 2. This result is consistent with the phase diagram in [23] as far as the stability region of 2-multichimera is concerned. However, in [23], the stationary and breathing 2-multichimeras are not distinguished, and the region of the breathing one is identified as a part of the region of the stationary one.

It is mostly assumed that the chimera state for Eq. (1) is a stationary state in the rotating frame with a frequency $\Omega$. This precisely means that the local mean field,

$$
Y(x, t):=\int_{-\pi}^{\pi} d y G(x-y) e^{i \theta(y, t)},
$$

acting on the oscillator located in point $x$, takes the form $Y(x, t)=\tilde{Y}(x) e^{i \Omega t}$. Then the global order parameter,

$$
Z(t):=\frac{1}{2 \pi} \int_{-\pi}^{\pi} d y e^{i \theta(y, t)}
$$

also takes the form $Z(t)=\tilde{Z} e^{i \Omega t}$. Here, $|Z(t)|$ denotes the synchronization degree of all oscillators, that is, all oscillators are completely synchronized in phase for $|Z(t)|=1$ and otherwise for $0 \leqslant|Z(t)|<1$. In the case of the stationary 2-multichimera as in Fig. 1, $|Z(t)|$ should vanish in the continuum limit $N \rightarrow \infty$, but we found that $|Z(t)|$ can oscillate periodically at appropriate parameters $(\alpha, r)$ and sufficiently large $N$. Figure 3 shows the time evolution of $|Z(t)|$ for 2-multichimeras with $N=100000$. The blue solid line ( $\alpha=$ 1.480 and $r=0.360$ ) exhibits a clear periodic oscillation, while the orange dashed line $(\alpha=1.480$ and $r=0.440)$ merely exhibits a small fluctuation around zero. We call the 


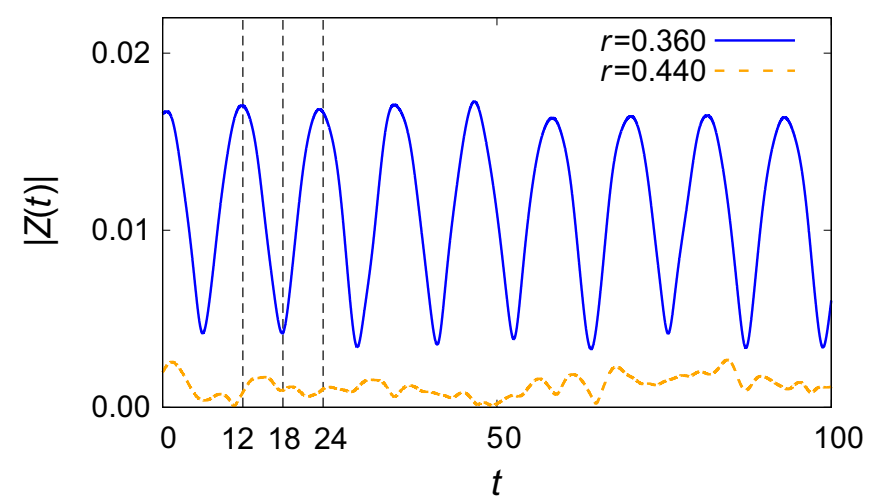

FIG. 3. Time evolution of the global order parameter $|Z(t)|$ for a 2-multichimera for Eq. (4) with $N=100000$ and $\alpha=1.480$. The 2-multichimera is breathing for $r=0.360$ (blue solid line), while it is stationary for $r=0.440$ (orange dashed line). Vertical dashed lines correspond to the times $t$ in Fig. 4.

former state breathing 2-multichimera, while we regard the latter as stationary 2-multichimera.

The detailed periodic behavior of the breathing 2-multichimera can be confirmed as the periodic oscillation of $|Y(x, t)|$ as shown in Fig. 4. $|Y(x, t)|$ takes a bimodal form, where the positions of the peaks correspond to each center of the coherent regions. Within a period of the global order parameter $|Z(t)|$ approximately corresponding to $t=12 \sim 24$ in Fig. 3, $|Y(x, t)|$ experiences the variation in one-half of its period, and within the next period of $|Z(t)|,|Y(x, t)|$ completes its whole period. Therefore, the period of $|Y(x, t)|$ is double that of $|Z(t)|$. In the simulation of Fig. 4, the angular frequency of $|Y(x, t)|$ is calculated as about 0.270 , which is compared with the result of the linear stability analysis in Sec. IV.

We can distinguish between stationary and breathing 2-multichimeras by studying the time evolution of $|Z(t)|$, but that is difficult for small $N$ because large fluctuation in $|Z(t)|$ is unavoidable. To distinguish between these clearly, we needed 10000 oscillators at least in our numerical simulation. Though such breathing properties of the standard chimera states in one-dimensional phase oscillators systems is observed by introducing phase lag parameter heterogeneity [10], we note that the present breathing 2-multichimera does not require such heterogeneity. In this paper, we focus on this breathing 2-multichimera, and study the bifurcation mechanism from the stationary 2-multichimera.

\section{STATIONARY 2-MULTICHIMERA}

Here, we study the basic properties of stationary 2-multichimeras. We first rewrite Eq. (1) as

$$
\dot{\theta}(x, t)=\omega+\operatorname{Im}\left[e^{-i \theta(x, t)} e^{-i \alpha} Y(x, t)\right] .
$$

Furthermore, we define the local order parameter [24],

$$
z(x, t):=\lim _{\delta \rightarrow 0+} \frac{1}{2 \delta} \int_{x-\delta}^{x+\delta} d y e^{i \theta(y, t)},
$$

and obtain $Y(x, t)=\int_{-\pi}^{\pi} d y G(x-y) z(y, t) .|z(x, t)|$ denotes the synchronization degree of oscillators around point $x$,
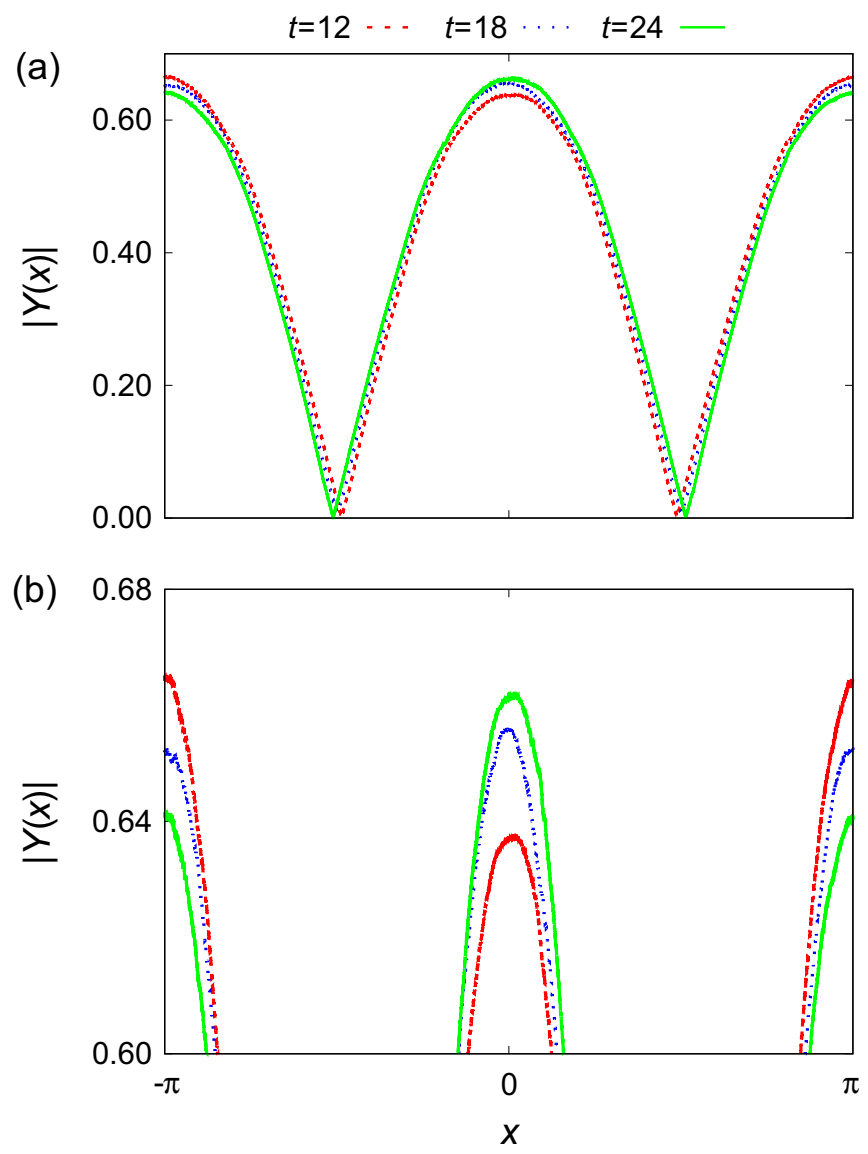

FIG. 4. Snapshot of the local mean field $|Y(x, t)|$ for the breathing 2-multichimera for Eq. (4) with $N=100000, \alpha=1.480$, and $r=$ 0.360 . (a) The global view of the snapshot; (b) the upper enlarged view. The red dashed line $(t=12)$ and green solid line $(t=24)$ lines approximately correspond to peaks of the global order parameter $|Z(t)|$, while the blue dotted line $(t=18)$ approximately corresponds to a valley, as shown in Fig. 3.

similarly to the global order parameter $|Z(t)|$. For $|z(x, t)|=$ 1 , the oscillators in the neighborhood of $x$ are completely synchronized in phase. Otherwise, when their phases are scattered, we obtain $0 \leqslant|z(x, t)|<1$. Therefore, we can identify $|z(x, t)|=1$ and $0 \leqslant|z(x, t)|<1$ as the coherent and incoherent regions for chimera states, respectively.

Following the method in $[9,13]$ using the WatanabeStrogatz approach [36], we can obtain the evolution equation of $z(x, t)$ as

$$
\dot{z}(x, t)=i \omega z(x, t)+\frac{1}{2} e^{-i \alpha} Y(x, t)-\frac{1}{2} e^{i \alpha} z^{2}(x, t) Y^{*}(x, t),
$$

using Eq. (8), where the symbol $*$ denotes the complex conjugate. Equation (10) can also be obtained by another method $[10,21]$ using the Ott-Antonsen ansatz $[37,38]$. Assuming the stationary solution $z(x, t)=\tilde{z}(x) e^{i \Omega t}$, Eq. (10) is rewritten as

$$
0=i \Delta \tilde{z}(x)+\frac{1}{2} e^{-i \alpha} \tilde{Y}(x)-\frac{1}{2} e^{i \alpha} \tilde{z}^{2}(x) \tilde{Y}^{*}(x),
$$

where $\Delta:=\omega-\Omega$. Solving Eq. (11) as a quadratic equation in terms of $\tilde{z}(x)$ and integrating the solution, we can obtain the 
self-consistency equation,

$$
\begin{gathered}
\tilde{Y}(x)=i e^{-i \alpha} \int_{-\pi}^{\pi} d y G(x-y) \tilde{Y}(y) h(y), \\
h(x):= \begin{cases}{\left[\Delta-\sqrt{\Delta^{2}-R(x)^{2}}\right] / R(x)^{2}} & {[\Delta>R(x)]} \\
{\left[\Delta-i \sqrt{R(x)^{2}-\Delta^{2}}\right] / R(x)^{2}} & {[\Delta \leqslant R(x)],}\end{cases}
\end{gathered}
$$

where $R(x) e^{i \Theta(x)}:=\tilde{Y}(x)=Y(x, t) e^{-i \Omega t}$. These equations correspond to the self-consistency equation derived by $\mathrm{Ku}-$ ramoto and Battogtokh [3]. Equations (12) and (13) are composed of two equations given by the real and imaginary parts, but have three real unknowns $R(x), \Theta(x)$, and $\Delta$. Therefore, we need to add the third condition to solve them. The third condition can be obtained from the fact that Eqs. (12) and (13) are invariant under any rotation, $\Theta(x) \rightarrow \Theta(x)+\Theta_{0}[5-7,24]$. Based on the above, we have chosen the condition

$$
\Theta(-\pi)=-\frac{\pi}{2} .
$$

Equations (12) and (13) under Eq. (14) can be numerically solved by the following iteration procedure [6,7]. First, we prepare an initial function $\tilde{Y}(x)$, i.e., $R(x)$ and $\Theta(x)$, and obtain $\Delta$ satisfying Eq. (14) from Eqs. (12) and (13) by Newton's method with respect to $\Delta$. Second, substituting $\tilde{Y}(x)$ and $\Delta$ into the right-hand side of Eq. (12), we generate a new $\tilde{Y}(x)$ from the left-hand side. Third, we obtain a new $\Delta$ satisfying Eq. (14), again by Newton's method, using the new $\tilde{Y}(x)$. It only remains to repeat the second and third steps until both $\tilde{Y}(x)$ and $\Delta$ converge. Note that space translational symmetry of $\tilde{Y}(x)$ is not eliminated in this iteration procedure, so the spatial position of $\tilde{Y}(x)$ depends on the initial condition.

According to [21], it is analytically proved that 2-multichimeras exist under the condition $g_{1} \neq 0$ for the coupling kernel given by Eq. (2), and the local mean field $\tilde{Y}(x)$ of a stationary 2-multichimera is given by an even function,

$$
\tilde{Y}(x)=\sum_{m=1}^{\infty} C_{2 m-1} \cos [(2 m-1) x],
$$

where $C_{2 m-1} \in \mathbb{C}$. This means that all 2-multichimera solutions of Eqs. (12) and (13) can be transformed into the form given by Eq. (15) by the appropriate spatial translation. Using Eq. (15), $h(x)$ turns out to be an even function because $R(x)=|\tilde{Y}(x)|$ is also even. Let a set of $\tilde{Y}(x)$ satisfying Eq. (15) and $\Delta$ be a solution of Eqs. (12) and (13). By substituting Eqs. (2) and (15) into Eqs. (12) and (13) and eliminating the terms whose integrands are odd functions of $y$, we obtain

$$
\begin{aligned}
\tilde{Y}(x)= & 2 i e^{-i \alpha} \sum_{k=0}^{\infty} g_{k} \cos (k x) \sum_{m=1}^{\infty} C_{2 m-1} \\
& \times \int_{0}^{\pi} d y \cos (k y) \cos [(2 m-1) y] h(y) .
\end{aligned}
$$

Changing the variable as $y^{\prime}=y-\pi / 2$ in the integration in Eq. (16), the function $h\left(y^{\prime}+\pi / 2\right)$ in the integrand is an even function of $y^{\prime}$ because of Eq. (15). We again eliminate the terms whose integrands are odd functions of $y^{\prime}$, and obtain

$$
\begin{aligned}
\tilde{Y}(x)= & 2 i e^{-i \alpha} \sum_{l=1}^{\infty} g_{2 l-1} \cos [(2 l-1) x] \sum_{m=1}^{\infty} C_{2 m-1}(-1)^{l+m} \\
& \times \int_{-\frac{\pi}{2}}^{\frac{\pi}{2}} d y^{\prime} \sin \left[(2 l-1) y^{\prime}\right] \sin \left[(2 m-1) y^{\prime}\right] h\left(y^{\prime}+\frac{\pi}{2}\right) .
\end{aligned}
$$

From the above, for $\tilde{Y}(x)$ and $\Delta$ of a stationary 2-multichimera satisfying Eqs. (12) and (13), we can finally obtain

$$
\tilde{Y}(x)=i e^{-i \alpha} \sum_{l=1}^{\infty} g_{2 l-1} \sum_{m=1}^{\infty} C_{2 m-1} A_{l m} \cos [(2 l-1) x],
$$

where $A_{l m}$ is a complex constant. Equation (18) shows that $\tilde{Y}(x)$ and $\Delta$ of a stationary 2-multichimera depends only on the odd harmonic coefficients $g_{2 m-1}$, not on the even harmonic coefficients $g_{2 m}$ of the coupling kernel $G(x)$. This is because we recover Eq. (18) even when we substitute the identical set of $\tilde{Y}(x)$ and $\Delta$ into Eqs. (12) and (13) with another coupling kernel, for example,

$$
G_{\text {odd }}(x)=\sum_{m=1}^{\infty} g_{2 m-1} \cos [(2 m-1) x],
$$

having the same set of odd harmonic coefficients $g_{2 m-1}$. Therefore, each stationary 2-multichimera for $G(x)$ and $G_{\text {odd }}(x)$ systems has an identical local mean field. This does not mean that the stability properties of these 2-multichimeras are also identical. However, Eqs. (12) and (13) for each system have an identical solution of the stationary 2-multichimera, whether or not each chimera is stable. This property of the coupling kernel $G_{\text {odd }}(x)$ is useful in the linear stability analysis for the stationary 2-multichimeras mentioned in Sec. IV.

To illustrate the above property for our step kernel given by Eq. (3), we performed a numerical simulation using a new coupling kernel $G_{\text {odd }}(x)$ with the same set of odd harmonic coefficients $g_{2 m-1}$ as for Eq. (3). By regarding Eq. (3) as a $2 \pi$-periodic function of $x, G_{\text {odd }}(x)$ can also be obtained as $G_{\text {odd }}(x)=[G(x)-G(x-\pi)] / 2$, which we used in the numerical simulation instead of the Fourier expansion in Eq. (19). Then, also for this $G_{\text {odd }}(x)$, we observed stationary 2-multichimeras. Figure 5 shows the time-averaged local mean fields $\langle\tilde{Y}(x)\rangle$ of the stationary 2-multichimeras using the step kernel $G(x)$ and the corresponding $G_{\text {odd }}(x)$. They are clearly identical and also agree with the numerical solution $\tilde{Y}(x)$ to the self-consistency equations given by Eqs. (12) and (13) with $G_{\text {odd }}(x)$. Note that the solid lines in Fig. 5 are obtained for $G_{\text {odd }}(x)$, not for $G(x)$. We tried to numerically solve Eqs. (12) and (13) with the step kernel $G(x)$, but we could not obtain a stationary solution $\tilde{Y}(x)$ of 2-multichimera because $\tilde{Y}(x)$ converged to another solution corresponding to a standard chimera state with one coherent and one incoherent region, under any initial conditions. Note that since where the iteration converges is attributed to the property of the iteration procedure, this does not mean that the stationary 2-multichimera solution does not exist for the $G(x)$ system. 

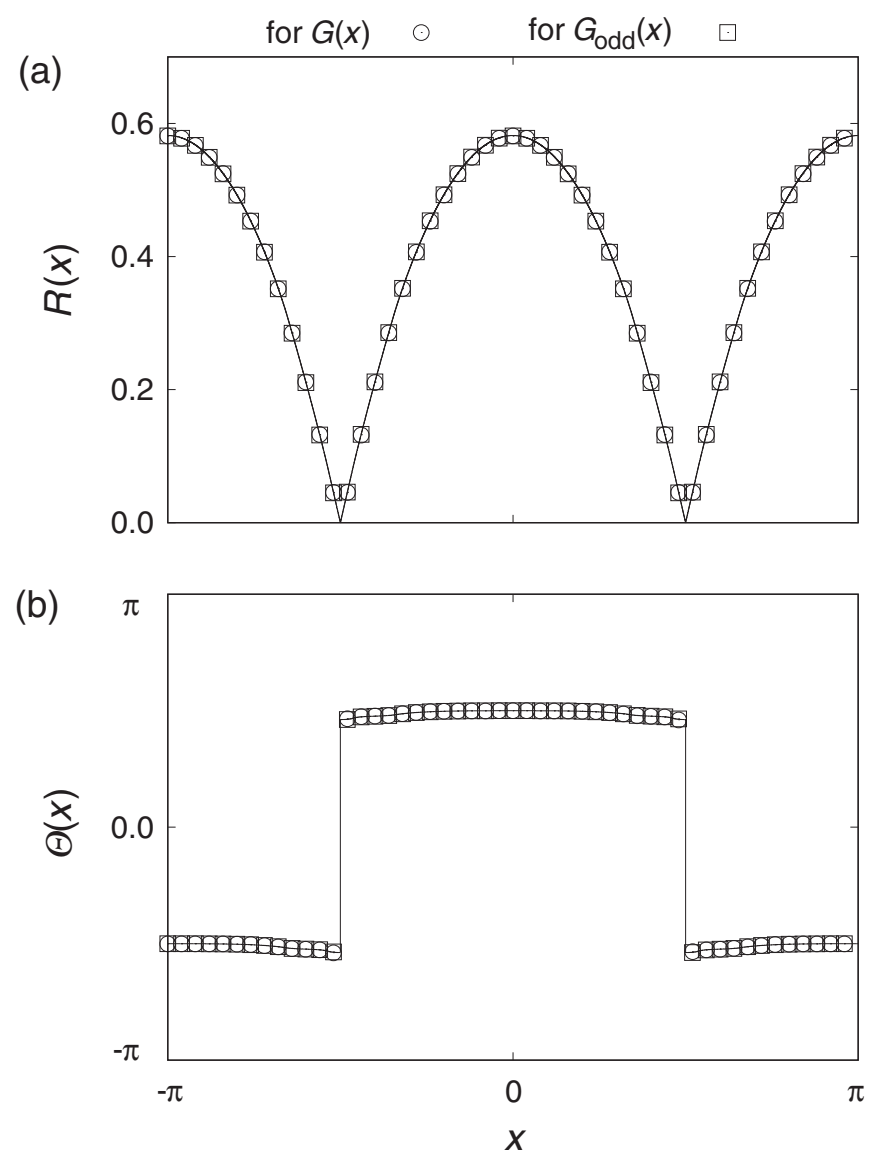

FIG. 5. Local mean field $\tilde{Y}(x)$ of a stationary 2-multichimera. (a) The amplitude $R(x)$; (b) the argument $\Theta(x)$. Open circles denote the time-averaged local mean field $\langle\tilde{Y}(x)\rangle$ for Eq. (1) with the step kernel $G(x)$, namely, Eq. (4), with $N=100000, \alpha=1.480$, and $r=0.440$, and open squares denote $\langle\tilde{Y}(x)\rangle$ for $G_{\text {odd }}(x)$ with the same parameters. Note that those are plotted once every 2000 oscillators. The solid line denotes the numerical solution $\tilde{Y}(x)$ to the self-consistency equations given by Eqs. (12) and (13) with $G_{\text {odd }}(x)$.

2-multichimeras can also appear for Eq. (1) with other $G_{\text {odd }}(x)$, e.g., $G_{\text {odd }}(x)=g_{1} \cos (x)$ [24], $G_{\text {odd }}(x)=$ $g_{1} \cos (x)+g_{3} \cos (3 x)$ as shown in Fig. 6, and so on. In our numerical simulations for various $G_{\text {odd }}(x)$ systems, we found an interesting property common to 2-multichimeras for $G_{\text {odd }}(x)$, which is an exact relationship between the phase $\theta(x, t)$ as

$$
|\theta(x, t)-\theta(x-\pi, t)|=\pi,
$$

on any point $x$. In fact, from Eq. (8) with any $G_{\text {odd }}(x)$, we obtain $\dot{\theta}(x, t)-\dot{\theta}(x-\pi, t)=0$ for Eq. (20) by using the relation $Y(x, t)=-Y(x-\pi, t)$ satisfied at any time. This implies that Eq. (20) can be a solution to Eq. (1) with $G_{\text {odd }}(x)$ whether stable or not, but our simulations show that the system with $G_{\text {odd }}(x)$ always converges to the solution given by Eq. (20) under any initial conditions. For the kernel other than $G_{\text {odd }}(x)$, this property given by Eq. (20) is not exact, but seems to be satisfied only in the meaning of average, as seen in Fig. 1(a).

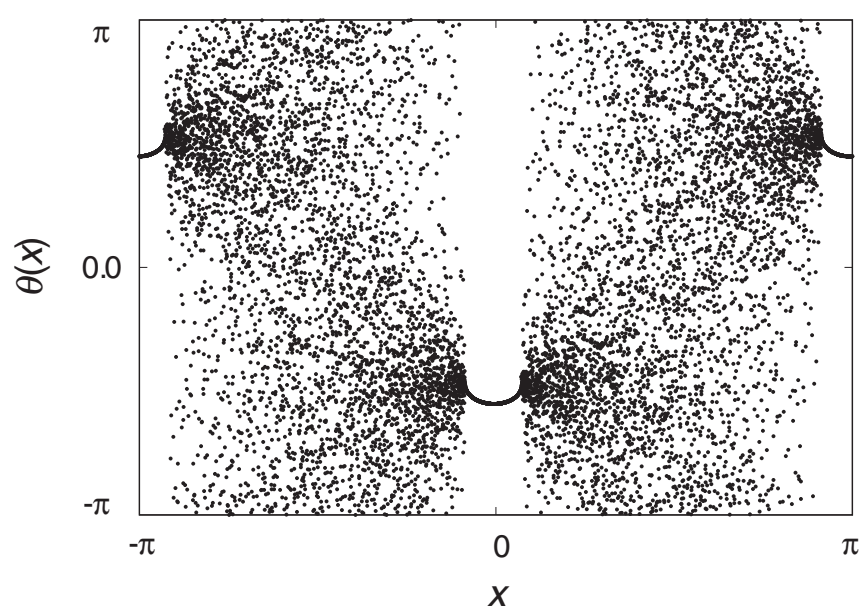

FIG. 6. Snapshot of a 2-multichimera for Eq. (1) with $G_{\text {odd }}(x)=$ $g_{1} \cos (x)+g_{3} \cos (3 x)$ with $N=10000, \alpha=1.500, g_{1}=1$, and $g_{3}=-0.0916$. The phase $\theta(x, t)$ on any point $x$ satisfies Eq. (20).

\section{BREATHING 2-MULTICHIMERA}

As described above, 2-multichimeras for Eq. (1) with the step kernel $G(x)$ do not satisfy Eq. (20). In our simulations, however, we often observed breathing 2-multichimeras instead of stationary 2-multichimeras, as shown in Fig. 3. This breathing 2-multichimera is characterized by the global order parameter $|Z(t)|$ oscillating periodically. Therefore, 2-multichimeras for $G_{\text {odd }}(x)$ satisfying Eq. (20) cannot breathe because the global order parameter of Eq. (20) exactly vanishes.

It is known that the breathing chimeras in the other studies $[8,10,32]$ branch via Hopf bifurcation from stable stationary chimeras. If the present breathing 2-multichimera also branches via Hopf bifurcation, an unstable stationary 2-multichimera should exist in the neighborhood of the bifurcation point. The local mean field of this unstable stationary 2-multichimera should be a solution to the self-consistency equations given by Eqs. (12) and (13), and identical with that of the stationary 2-multichimera for the $G_{\text {odd }}(x)$ system.

In order to investigate this bifurcation, we analyze the linear stability of stationary 2-multichimeras. By substituting $z(x, t)=[\tilde{z}(x)+v(x, t)] e^{i \Omega t}$ with the stationary solution $\tilde{z}(x) e^{i \Omega t}$ and a small perturbation $v(x, t)$ into Eq. (10), we obtain a linear evolution equation for $v(x, t)$,

$$
\begin{array}{r}
\dot{v}(x, t)=g(x) \tilde{z}(x)+\frac{1}{2} e^{-i \alpha} V(x, t)-\frac{1}{2} e^{i \alpha} \tilde{z}^{2}(x) V \\
g(x):= \begin{cases}i \sqrt{\Delta^{2}-R(x)^{2}} & {[\Delta>R(x)]} \\
-\sqrt{R(x)^{2}-\Delta^{2}} & {[\Delta \leqslant R(x)],}\end{cases} \\
V(x, t):=\int_{-\pi}^{\pi} d y G(x-y) v(y, t),
\end{array}
$$

where $\Delta=\omega-\Omega$. We rewrite Eqs. (21)-(23) as $\dot{\boldsymbol{v}}=L \boldsymbol{v}$ using $\boldsymbol{v}(x, t)=[\operatorname{Re} v(x, t), \operatorname{Im} v(x, t)]^{T}$ and solve the eigenvalue problem of $L$. According to [21,24], the spectrum of $L$ consists of the essential spectrum and the point spectrum. In the present case, the essential spectrum is given by $g(x)$ consisting of pure imaginary and negative real eigenvalues, which correspond to incoherent and coherent regions, respectively. Therefore, the 

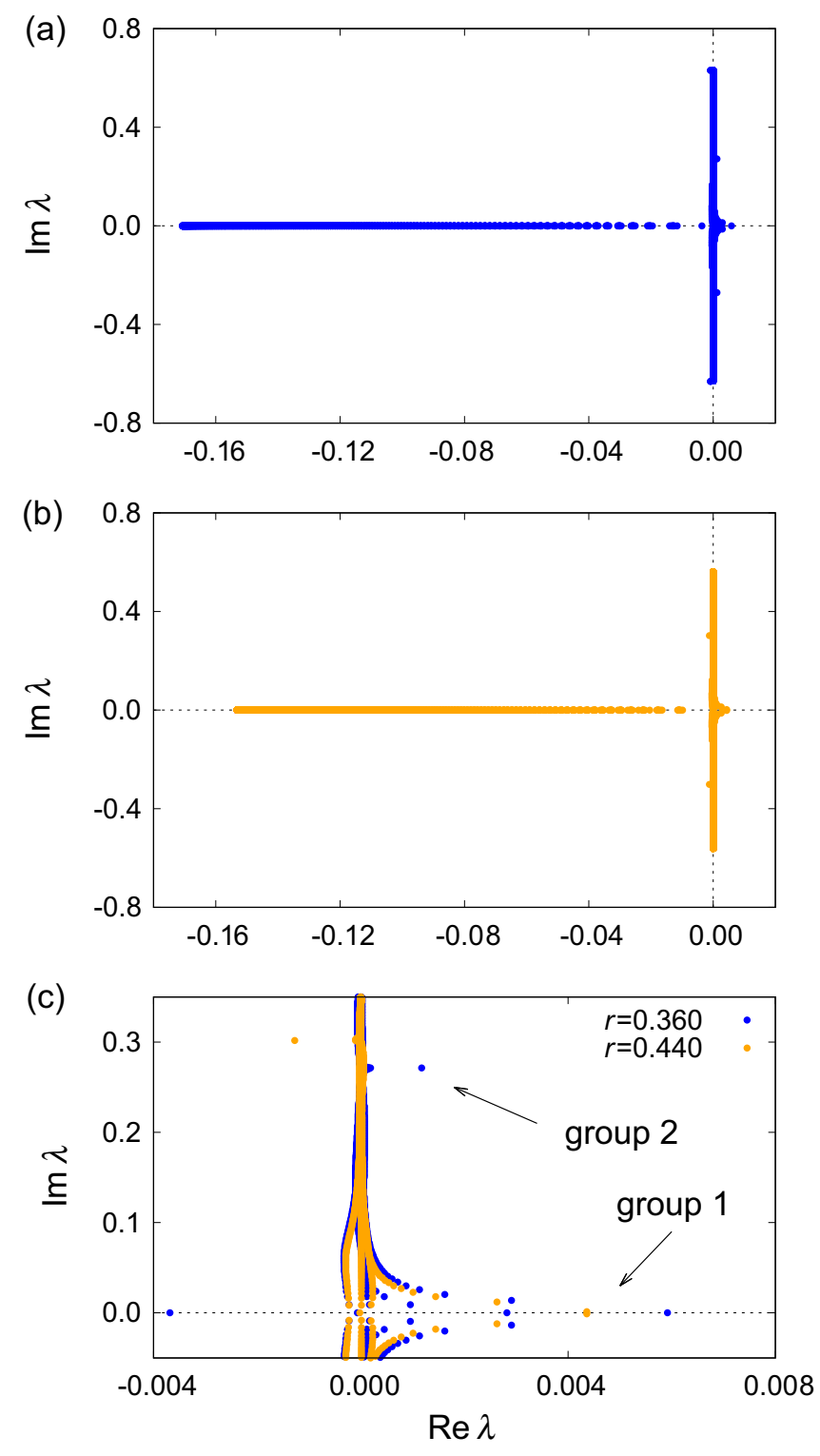

FIG. 7. Complex eigenvalues $\lambda$ of $L_{d}$ with $M=5000$ and $\alpha=$ 1.480 using $\tilde{Y}(x)$ with $N=200000$. (a) All eigenvalues for the unstable stationary 2-multichimera that changes to a breathing one $(r=0.360)$; (b) those for the stable stationary 2-multichimera $(r=$ 0.440 ). (c) The enlarged view of (a) and (b) denoted by the blue and orange points, respectively. The dashed lines in each panel are drawn only for reference.

stability of stationary 2-multichimeras should be determined only by the point spectrum.

If the number of nonzero $g_{k}$ in Eq. (2) is finite, we may solve the eigenvalue problem of a finite size matrix to obtain the point spectrum [21,24]. However, the step kernel given by Eq. (3) has infinite numbers of nonzero $g_{k}$. Therefore, we discretize the space coordinate $x \rightarrow x_{j}=-\pi+2 \pi j / M(j=0, \ldots$, $M-1)$ and compute all eigenvalues $\lambda$ by solving the eigenvalue problem of $2 M \times 2 M$ matrix $L_{d}$ such that $\dot{\boldsymbol{v}}_{d}=L_{d} \boldsymbol{v}_{d}$ with $\boldsymbol{v}_{d}(t)=\left[\ldots, \operatorname{Re} v\left(x_{j}, t\right), \operatorname{Im} v\left(x_{j}, t\right), \ldots\right]^{T}$ [33,39]. In order to solve this problem, we first need to prepare $\tilde{Y}(x)$ and $\Delta$ of the stationary 2-multichimera for Eq. (4) numerically, but could not obtain them by solving

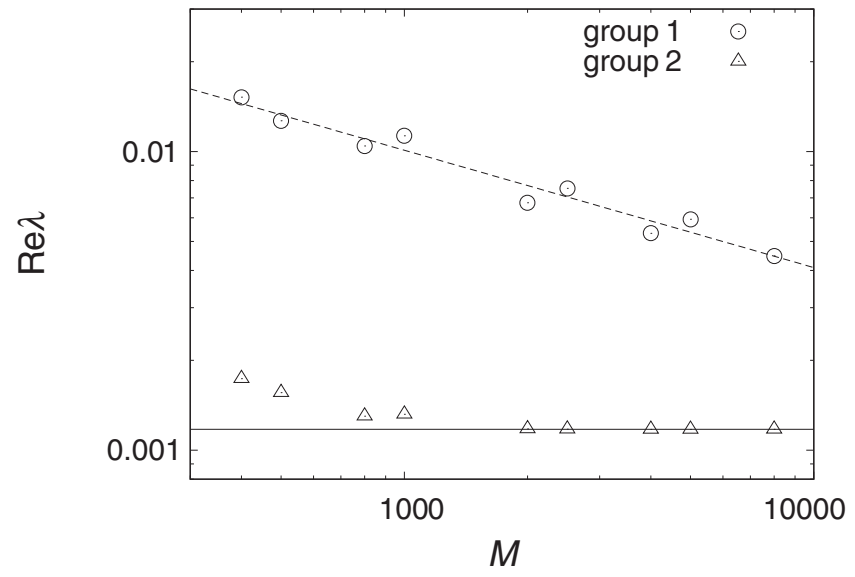

FIG. 8. Transition of the positive real parts of the eigenvalues of $L_{d}$ for an unstable stationary 2-multichimera ( $\alpha=1.480$ and $r=$ 0.360 ) with increasing $M$. Open circles denote the maximum values of the real parts of the eigenvalues in group 1 , and open triangles denote those in group 2. The data for group 1 are fitted linearly (dashed line) in the log-log plot, and go to zero with increasing $M$. In contrast, the data for group 2 converge to a positive constant $1.175 \times 10^{-3}$ (solid line).

the self-consistency equations given by Eqs. (12) and (13) with the step kernel $G(x)$ because $\tilde{Y}(x)$ converged to another solution by the iteration procedure, as mentioned in Sec. III. We accordingly used $\tilde{Y}(x)$ and $\Delta$ of Eqs. (12) and (13) with the corresponding $G_{\text {odd }}(x)$, instead of $G(x)$. Although $G_{\text {odd }}(x)$ is used for computing $\tilde{Y}(x)$ and $\Delta$, we note that we insert the original kernel $G(x)$ into Eqs. (21)-(23) to solve the eigenvalue problem. Figure 7(a) shows all the eigenvalues $\lambda$ of $L_{d}$ with $M=5000, \alpha=1.480$, and $r=0.360$ on the complex plane. As seen from the figure, we have some eigenvalues with positive real part, because the stationary 2-multichimera is unstable and changes into a breathing one at these parameters. We can regard those eigenvalues as roughly separating into two groups. Group 1 consists of some eigenvalues around the real axis and group 2 consists of others around the imaginary values about 0.270 and their complex conjugate, as shown in Fig. 7(c).

Even though we can observe the eigenvalues with a positive real part, we cannot easily tell whether they belong to the point spectrum or a fluctuation of the essential spectrum caused by finite discretization. If an eigenvalue with a positive real part belongs to such a fluctuation, its real part should go to zero in the continuum limit $M \rightarrow \infty$, while an eigenvalue in the point spectrum keeps the positive real part in that limit. We computed the eigenvalues of $L_{d}$ with various $M$ and found their limiting behaviors as $M$ is increased, as shown in Fig. 8 .

From Fig. 8, we can see that the maximum value of the real parts of the eigenvalues in group 1 tends to go to zero, while that value in group 2 converges to a positive constant. Therefore, it turns out that at least a pair of the complex conjugate eigenvalues in group 2 belongs to the point spectrum, while the eigenvalues in group 1 belong to the fluctuation of the essential spectrum by finite discretization. At the other parameters where the stationary 2-multichimera is stable, the point spectrum contains only the eigenvalues with a negative real part, as shown in Fig. 7(b). 


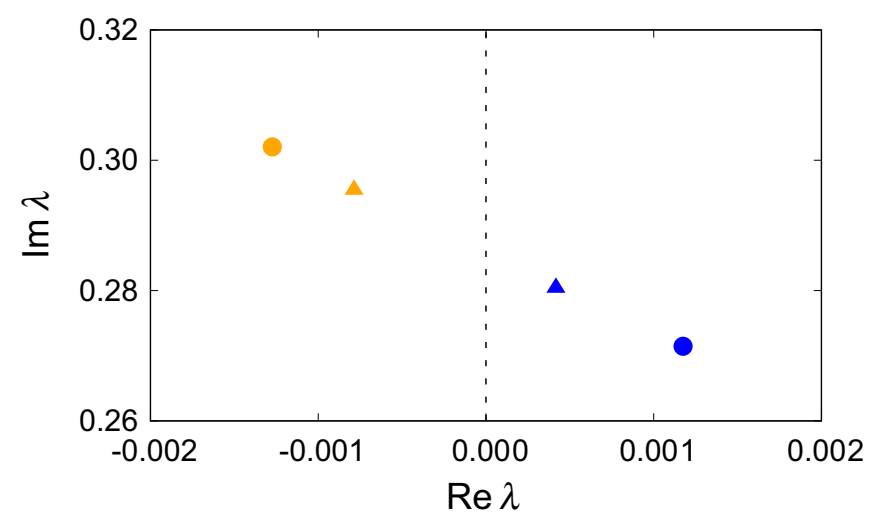

FIG. 9. Hopf bifurcation for fixed $\alpha=1.480$. Each point shows the eigenvalue with the positive imaginary part and the maximum absolute value of the real part in the point spectrum for $r=0.440$ (orange circle), $r=0.420$ (orange triangle), $r=0.380$ (blue triangle), and $r=0.360$ (blue circle), and corresponds to the black solid line in Fig. 2. The point for $r=0.400$ is omitted in the figure because we could not distinguish the point spectrum from other eigenvalues for $r=0.400$ that is very close to the Hopf bifurcation point. The dashed line is the imaginary axis.

Figure 9 shows that a Hopf bifurcation from a stationary 2-multichimera to a breathing one occurs for $\alpha=1.480$, denoted by the vertical black solid line in Fig. 2. The Hopf bifurcation points for $\alpha=1.480$ and other values are shown as the blue line in Fig. 2. However, we could not determine the bifurcation points for $\alpha$ close to $\pi / 2$ because it is difficult to distinguish the point spectrum whose real parts are almost zero for those $\alpha$. We note that the absolute values of the imaginary parts of the point spectrum, as shown in Fig. 7(a), are nearly equal to the angular frequency of the local mean field $|Y(x, t)|$, as shown in Fig. 4, which is calculated to be about 0.270 .
This result agrees with the occurrence of a supercritical Hopf bifurcation.

\section{SUMMARY}

We studied 2-multichimera with two coherent and incoherent regions in one-dimensional nonlocally coupled phase oscillators described as Eq. (1). First, we showed that the local mean field $\tilde{Y}(x)$ of stationary 2-multichimeras depends on only odd harmonic coefficients $g_{2 m-1}$ of the coupling kernel function $G(x)$. This implies that if $G(x)$ has the same set of the odd harmonic coefficients, then $\tilde{Y}(x)$ of stationary 2-multichimeras are common to all those $G(x)$ systems for the same parameters. We could actually apply $\tilde{Y}(x)$ of the $G_{\text {odd }}(x)$ system to the linear stability analysis of stationary 2-multichimeras for the $G(x)$ system, even though we could not obtain $\tilde{Y}(x)$ of stationary 2-multichimeras for the $G(x)$ system. The method used in this paper is based on the fact that $\tilde{Y}(x)$ of stationary 2-multichimeras is characterized by only odd harmonic components, namely, Eq. (15). We expect that a similar method is applied to other stationary multichimera states because their local mean fields are also characterized by a set of specific harmonic components [21].

Next, we numerically found that breathing 2-multichimeras with oscillatory global order parameter $|Z(t)|$ appear for Eq. (1) with the step kernel given by Eq. (3) without introducing parameter heterogeneity [10]. Moreover, we clarified that the system exhibits a Hopf bifurcation from a stationary 2-multichimera to a breathing one by the linear stability analysis for the stationary 2-multichimera. In contrast to the $G(x)$ system, 2-multichimeras for $G_{\text {odd }}(x)$ cannot breathe because the system converges to the solution given by Eq. (20) with vanishing $|Z(t)|$. Therefore, it is inferred that the coupling kernel is an important factor for the appearance of breathing chimeras in the one-dimensional system. It may be interesting to find other breathing chimeras by using the appropriate coupling kernel similarly, but it is an open problem.
[1] Y. Kuramoto, Chemical Oscillation, Waves, and Turbulence (Springer, Berlin, 1984).

[2] A. Pikovsky, M. Rosenblum, and J. Kurths, Synchronization: A Universal Concept in Nonlinear Sciences (Cambridge University Press, Cambridge, 2003).

[3] Y. Kuramoto and D. Battogtokh, Nonlinear Phenom. Complex Syst. 5, 380 (2002).

[4] S.-i. Shima and Y. Kuramoto, Phys. Rev. E 69, 036213 (2004).

[5] D. M. Abrams and S. H. Strogatz, Phys. Rev. Lett. 93, 174102 (2004).

[6] D. M. Abrams and S. H. Strogatz, Int. J. Bifurc. Chaos 16, 21 (2006).

[7] G. C. Sethia, A. Sen, and F. M. Atay, Phys. Rev. Lett. 100, 144102 (2008).

[8] D. M. Abrams, R. Mirollo, S. H. Strogatz, and D. A. Wiley, Phys. Rev. Lett. 101, 084103 (2008).

[9] A. Pikovsky and M. Rosenblum, Phys. Rev. Lett. 101, 264103 (2008).

[10] C. R. Laing, Physica D 238, 1569 (2009).

[11] E. A. Martens, C. R. Laing, and S. H. Strogatz, Phys. Rev. Lett. 104, 044101 (2010).
[12] O. E. Omel'chenko, M. Wolfrum, and Y. L. Maistrenko, Phys. Rev. E 81, 065201 (2010).

[13] M. Wolfrum, O. E. Omel'chenko, S. Yanchuk, and Y. L. Maistrenko, Chaos 21, 013112 (2011).

[14] I. Omelchenko, Y. Maistrenko, P. Hövel, and E. Schöll, Phys. Rev. Lett. 106, 234102 (2011).

[15] M. Wolfrum and O. E. Omel'chenko, Phys. Rev. E 84, 015201 (2011).

[16] I. Omelchenko, B. Riemenschneider, P. Hövel, Y. Maistrenko, and E. Schöll, Phys. Rev. E 85, 026212 (2012).

[17] M. R. Tinsley, S. Nkomo, and K. Showalter, Nat. Phys. 8, 662 (2012).

[18] A. M. Hagerstrom, T. E. Murphy, R. Roy, P. Hövel, I. Omelchenko, and E. Schöll, Nat. Phys. 8, 658 (2012).

[19] E. A. Martens, S. Thutupalli, A. Fourriére, and O. Hallatschek, Proc. Natl. Acad. Sci. USA 110, 10563 (2013).

[20] I. Omelchenko, O. E. Omel'chenko, P. Hövel, and E. Schöll, Phys. Rev. Lett. 110, 224101 (2013).

[21] O. E. Omel'chenko, Nonlinearity 26, 2469 (2013).

[22] L. Schmidt, K. Schönleber, K. Krischer, and V. García-Morales, Chaos 24, 013102 (2014). 
[23] Y. L. Maistrenko, A. Vasylenko, O. Sudakov, R. Levchenko, and V. L. Maistrenko, Int. J. Bifurc. Chaos 24, 1440014 (2014).

[24] J. Xie, E. Knobloch, and H.-C. Kao, Phys. Rev. E 90, 022919 (2014).

[25] D. P. Rosin, D. Rontani, N. D. Haynes, E. Schöll, and D. J. Gauthier, Phys. Rev. E 90, 030902 (2014).

[26] P. Ashwin and O. Burylko, Chaos 25, 013106 (2015).

[27] M. J. Panaggio and D. M. Abrams, Nonlinearity 28, R67 (2015).

[28] S. W. Haugland, L. Schmidt, and K. Krischer, Sci. Rep. 5, 9883 (2015).

[29] L. Schmidt and K. Krischer, Chaos 25, 064401 (2015).

[30] I. Omelchenko, A. Zakharova, P. Hövel, J. Siebert, and E. Schöll, Chaos 25, 083104 (2015).
[31] Y. Suda and K. Okuda, Phys. Rev. E 92, 060901 (2015).

[32] M. J. Panaggio, D. M. Abrams, P. Ashwin, and C. R. Laing, Phys. Rev. E 93, 012218 (2016).

[33] L. Smirnov, G. Osipov, and A. Pikovsky, J. Phys. A 50, 08LT01 (2017).

[34] H. Cheng, Q. Dai, N. Wu, Y. Feng, H. Li, and J. Yang, Commun. Nonlinear Sci. Numer. Simul. 56, 1 (2018).

[35] H. Sakaguchi and Y. Kuramoto, Prog. Theor. Phys. 76, 576 (1986).

[36] S. Watanabe and S. H. Strogatz, Physica D 74, 197 (1994).

[37] E. Ott and T. M. Antonsen, Chaos 18, 037113 (2008).

[38] E. Ott and T. M. Antonsen, Chaos 19, 023117 (2009).

[39] X. Zhang, A. Pikovsky, and Z. Liu, Sci. Rep. 7, 2104 (2017). 